\title{
Interactive comment on "Response of carbon and water fluxes to environmental variability in two Eastern North American forests of similar-age but contrasting leaf-retention and shape strategies" by Eric R. Beamesderfer et al.
}

Anonymous Referee \#2

Received and published: 22 February 2020

Reviewer \#2 recommendation: Return to author for major revisions

General comments

Carbon and water fluxes and their relationships with several environmental variables were presented in this study. The data were collected during the period from 20122017 at a conifer and a deciduous forest, which I believe represents an impressive dataset for both forest types. The presented and discussed results showed interesting findings in terms of responses of different forest types to drought and high tempera-

Printer-friendly version

Discussion paper 
ture. If there is a weakness, it is my opinion that it relates to the quantification and identification of the key environmental controls on the fluxes. A significant amount of the manuscript content is dedicated to describing the linear relationships between the fluxes and a series of meteorological variables. However, little regarding the physiological and physical processes is described. In addition, the results and discussions are a bit overwhelming and hard for me to find focuses, which makes the paper seem to lack a truly novel finding. In my opinion, this manuscript should be revised with focuses on: 1) outlining the key/novel science questions; and 2) emphasizing the findings that have clear/great implications in forest management and in ecosystem's responses to future climate. I believe this concern can be addressed through modest revision.

\section{Specific comments}

1.-Titile. The leaf-retention and shape strategies are only implied not studied in the manuscript. I suggest changing to a more relevant and accurate title.

2.-Line 16-24. The influences of drought and temperature on NEP and ET are entangled together here, which is a bit unclear. Also, some sentences seem to be repetitive. I suggest rewriting this part of the abstract to make it clearer.

3.-Abstract. Clarify and quantify (if possible) "greatly controlled", "greatly reduced", and "greatly impact".

4.-Line 55-57. Can you add a sentence or two summarizing the previous studies contrasting fluexes coniferous and deciduous forests?

5.-Methods. I noticed the distances of EC relative to the canopy top are different for the two sites. Would the heights of the EC affect the fluxes due to flux divergence or convergence?

Printer-friendly version

6.-Line 57. Is friction velocity a good metric for filtering intermittent turbulence? Previous studies show intermittent turbulence is frequently observed during evening hours at forested sites.

Discussion paper 
7.-Section 2.3. Have the data been filtered for stationarity?

8.-Section 2.3. The threshold $u^{*}$ seem to be large $(0.2$ or $0.3 \mathrm{~m} / \mathrm{s}$ are pretty standard)? Any explanations associated with the sites?

9.-Section 2.3. Add one or two sentences explaining how you processed/averaged the meteorological data.

10.-Section 2.4. Can you describe the uncertainties associated with the approach estimating phenological seasons?

11.-Line 257 and Line 349. Clarify "responded similarly".

12.-Line 255-262. Can you also show the standard deviations of the annual mean Ta in Fig.1?

13.-Line 265. Better explanation for the discrepancies is needed here. The discrepancies are over 300 umol m-2day-1 in spring. Is it in the range of the measurement uncertainty? l'd suggest check the downward PAR to tease out the influences from the canopies and to evaluate the meteorological differences.

14.-Line 267. Clarify "APAR was similar throughout the year". What are the values (mean and standard deviations) of the FPAR mentioned?

15.-Line 281. "Ts $(5 \mathrm{~cm})$ at TP39 exceeded that of TPD" seems to suggest that the PARgroud at TPD is less, which implies that the APAR at TPD should be higher in summer and autumn. Please explain.

16.-Line 296. Can you explain why 6-year mean day of season growth was used instead of the days of individual years?

17.-Line 327. Could you also add a sentence or two at the beginning of this paragraph to explain the physical meaning of the cumulative (seasonal and annual) fluxes, especially its differences from daily fluxes?

Printer-friendly version

Discussion paper 
18.-Line 336. "spring was the only season when daily GEP was similar between the forests". As shown in Table 3, the seasonal GEP in spring show larger differences between sites, which I think to some extent contradicts with your statement in Line 336 . Please reconcile. Also, when you compare the daily GEP for phenological seasons, how did you address the different lengths of the seasons (i.e. the different number of data points)?

19.-There are a few places where I have similar comments as the previous one. I suggest adding some explanations for the statistical techniques (ANOVA and MANOVA) you used, which would shed some light on the discrepancies. -Line 338. The cumulative GEP in autumn (and 2012, 2014, 2015 summer) is higher at TP39 (except for 2012). Does it contradict the argument in Line 338? -Line 352. "RE was higher at TPD". But the cumulative RE were lower at TPD in spring and autumn. -Line 384. Seasonal ET is more different in spring not autumn. Also, "daily ET" or "seasonal ET"?

20.-Line 339. "the 2016 summer was the only period ...". Clarify "sufficiently". Also, it seems a false statement to me because summer GEP in 2013 and 2017 are also greater at TPD.

21.-Line 353. Any figure or data to support this statement?

22.-Line 399. How the low WUE in winter is reflected in Figure 6c? Did you only use data from spring to autumn? If so, clarify in the manuscript.

23.-Line 405. Can you clarify "similar results"? The LUE at TPD is $30 \%$ higher than that at TP39.

24.-Line 406. Is the annual and seasonal LUE shown in the manuscript? If not, clarify it in the manuscript by adding "(data not shown)". Also, as shown in Table 3, TPD has lower annual GEP, which contradicts with the "greater GEP" referred here. Reconcile.

25.-Line 435. Do you mean "deciduous forest" instead of "conifer"? If not, add the correlation of annual NEP and summer RE for the conifer forest to Table 4 . If the

Printer-friendly version

Discussion paper 
answer is yes, l'd suggest delete this sentence because it conveys the same meaning as the following two sentences.

26.-Line 434-435. Can you add a brief explanation for the relationship of RE and spring Ta.

27.-Line 439-448. The annual GEP has no significant relationships with meteorological variables as stated in Line 425. But this paragraph talks about GEP and meteo controls. Is it only summer GEP discussed in this paragraph?

28.-Line 439. What does "flux parameterizations" mean here? Is it explained in the methodology section? If not, I suggest adding it to the methods section.

29.-Line 578. Is the assumption of similar carbon assimilation valid here given the different NEP?

30.-Table 3. Why the GEP sum for Jan 1 to SOS is missing? They seem to be available in Fig. 3.

31.-Table 4. Can you change this table to a figure similar to Fig. 4? The reasons are (i) you'd be able to show the standard deviations; (ii) the positive/negative correlation would be easier to tell.

32.-Table 5. What model did you use for this calculation?

33.-I notice the uncertainty analysis for measurements and calculations is missing. Can you add a brief subsection to Methods section (or wherever you find appropriate) dedicated to uncertainties?

\section{Minor comments}

1.-. I suggest changing all "warm temperatures/Ta" to "high temperatures/Ta" in the manuscript.

2.-Line 78. Clarify "controls". Environmental/meteorological controls? 
3.-Line 88-91. Are percentages available for the tree species?

4.-Line 119. Did you use the momentum and heat fluxes in this study? If not, there's no need to mention them.

5.-Line 258. What is the value of "record Ta". Also, "record high Ta".

6.-Line 315. Are the "days 230 to 290" 6-year mean? Explain.

7.-Line 325-326. This statement is not clear. Clarify or delete.

8.-Line 347. Define "outlier".

9.-Line 398. Clarify "the ratio of monthly ET". Then modify the figure caption accordingly.

10.-Line 354-355. Confusing sentence. How do "comparable" results shape the "differences"? Rephrase.

11.-Line 363. "for either site"? It's hard to tell that the monthly NEP is negative at TPD in Figure 5b. Rephrase.

12.-Line 416. $P$ value for being "significant"? "linear relationships of monthly Ta and monthly VPD"?

13.-Be concise. See examples below. -Line 325. "at first glance" is not necessary. -Line 341-342. "significant daily minimums and maximums" seems to be repetitive as "highly variable". -Line 417. Delete ",". -Line 409-410. Delete "and". Also, make the sentence more clear. -Line 372. "the highest" —-> "highest".

14. Given the different time scales used here, I suggest be more mindful about the uses of "daily, season, annual" when talking about fluxes. -Line 261. In "Ta at both sites", do you mean "daily mean Ta"? -Line 360. Change "The NEP in the conifer..." to "The annual NEP" or "The cumulative NEP". -Line 352. "spring and autumn RE was higher ...". Do you mean "daily RE in spring and summer"? -Line 410. Delete "When

Printer-friendly version

Discussion paper 
first considering ...". Change "ET"--> "Annual ET". -Line 325. Should "daily patterns" be "seasonal patterns"? Also, subsitute "expanded upon in Table3" with "the cumulative fluxes in Table 3", just to be clear and accurate.

15. I noticed quite a few miscitation or misspelling or inaccurate statements. See some examples below. -Line 270. "daily reductions in PAR (shouldn't it be APAR?)". -Line 401. $4.7 \longrightarrow 3.82 \mathrm{gC} \mathrm{kg}-1 \mathrm{H} 2 \mathrm{O}$. -Line 406. $\mathrm{R} 2=0.96 \longrightarrow \mathrm{R} 2=0.86$. -Line 535. "increases" _-> "decreases"? -Line 538. "most years" _-> "half of the years"? -Line 553. "during drought years" is not accurate. It's really just 2016.

16. I have a few minor comments regarding the tables and figures. See below. -Table 3. Can you highlight the highest and lowest annual fluxes with colored boxes? -Be more clear with figure captions, especially for words like "daily, monthly, seasonal, and annual". For example, "A daily time series" in Fig. 2 is a bit confusing. -Figure 3. Green-red combination is not color-blind friendly. Also, can you annotate SOS, EOG, SOB, and EDS on the top panels? -Figure 4 caption. Two "and".

Interactive comment on Biogeosciences Discuss., https://doi.org/10.5194/bg-2019-457, 2020. 